Calidad y medio ambiente Quality and Environment 



\title{
Un índice de capacidad de procesos para distribuciones multivariadas normales de variables correlacionadas y no correlacionadas
}

\author{
Erasmo Rafael Salazar* \\ Universidad de Oriente, Núcleo de Sucre. Venezuela \\ José Simón Fermín* \\ Instituto Universitario de Tecnología. Edo. Sucre, Venezuela \\ Recibido: 30 de mayo del 2016 / Aprobado: 23 de agosto del 2016
}

RESUmen: En el análisis de capacidad multivariado existen muchos aspectos aún no resueltos en torno a algunos índices y su aplicación. Por ejemplo, cuando las variables de calidad están correlacionadas y cuando no están correlacionadas. En este trabajo pretendemos proponer la construcción de un índice de capacidad multivariado que funcione para los dos casos anteriores, y presentar un ejemplo de aplicación de esta alternativa sobre un conjunto de datos reales. Nuestro índice obtuvo un amplio desempeño frente a otros índices de capacidad similares.

Palabras clave: análisis multivariado / indice de capacidad de procesos / indices de capacidad univariado / distribución normal

An index of capacity of processes for multivariate normal distributions of variables with correlation and without correlation

ABSTRACT: In the multivariate analysis of capacity many aspects even not exist resolved around some indexes and their application. For example, when the variables of quality are correlated and when they are not correlated. In this work we seek to propose the construction of a multivariate index of capacity that works for both previous cases, and to present an example of application of this alternative on a real dataset. A wide acting of our index is obtained proposed in front of other similar indexes of capacity.

Keywords: multivariateanalysis / process capability index / indexes of capacity univariate / normal distribution

* Correos electrónicos: eramo10464407@gmail.com, simon.fermin@gmail.com 


\section{INTRODUCCIÓN}

Los estudios de los índices de capacidad de procesos (ICP) se han desarrollado en el campo univariado y multivariado. Son estimaciones numéricas de la capacidad del proceso, es decir, nos dan una idea de cuán capaz es el proceso: a qué nivel cumple con las especificaciones. Estos estadísticos son muy útiles, ya que, además de ser fáciles de calcular, no tienen unidades de medida, por lo que permiten comparar distintos procesos. Básicamente son el cociente entre la amplitud tolerable del proceso (la distancia entre los límites de tolerancia o límites de especificación) y la amplitud real o natural del proceso; recordemos que, habitualmente, la distancia entre los límites de control es de 6 sigma.

Algunos de estos estadísticos se definen a partir de la media del proceso o del objetivo. Los índices de capacidad asociados con la variación a corto plazo son: $\mathrm{C}_{\mathrm{p}}, \mathrm{C}_{\mathrm{pk}}$, CPU y CPL. Por otro lado, los asociados con la variación a largo plazo son: $\mathrm{P}_{\mathrm{p}}, \mathrm{P}_{\mathrm{pk}}, \mathrm{PPU}, \mathrm{PPL} \mathrm{y} \mathrm{C}_{\mathrm{pm}}$. En la práctica, se suele considerar que 1,33 es el valor mínimo aceptable para un índice de capacidad, es decir, cualquier valor por debajo de esta cifra indicaría que, aunque esté bajo control estadístico, el proceso no cumple con las especificaciones deseadas. Dichos índices se usan bajo el supuesto de que el valor de las mediciones de las variables de calidad representa valores de variables aleatorias independientes con distribución normal y que el proceso se encuentra en el control estadístico, como lo establece Montgomery (2009).

Muchos autores realizan estudios de ICP considerando una sola variable; sin embargo, la gran mayoría de estos estudios abarca más de una variable. En los estudios de índices de capacidad de dominio multivariado también se han propuesto algunos índices de capacidad, que son extensiones de sus contrapartes univariado. Como señala Foster (et al., 2005), aún no hay una metodología que sea consistente para calcular índices de capacidad multivariados (ICPM), por lo tanto, hasta la fecha no existe consenso sobre el uso de un índice en particular. De aquí se desprende la necesidad de evaluar la capacidad de un proceso que tiene muchas variables correlacionadas y no correlacionadas, aunque resulta complejo de calcular y comprender.

Las investigaciones sobre índices de capacidad multivariados empezaron a realizarse a principios de 1990, pero no fue hasta el año 2005 cuando el número de publicaciones se incrementó. Desde entonces, diferentes ICPM han sido presentados. Estos ICPM se pueden dividir en 
cuatro grupos diferentes, como lo sugieren Shinde y Khadse (2009). Los grupos son:

- Grupo 1: basado en la razón de una región de tolerancia y una región del proceso. Ver como ejemplos a Taam y Liddy (1993), Shahriari y Abdollahzaleh (2009) y Pan y Lee (2010).

- Grupo 2: se basa en la probabilidad de producto de no conformidad. Un ejemplo de este grupo se puede encontrar en Castagliola y Castellanos (2005).

- Grupo 3: basado en el análisis de componentes principales. Ver como ejemplo a Wang y Chen (1998).

- Grupo 4: llamado otros (Shahriari y Lawrence, 1995).

En la tabla 1 se muestra un resumen de los ICPM que se encuentran en la literatura desde 1991 hasta el 2014. Se puede observar que la mayoría de los índices propuestos requieren de una distribución normal multivariada y también que la mayoría de ellos pertenecen al primer grupo. En la tabla, la columna DNM significa que el cálculo del índice propuesto requiere o no de una distribución normal multivariada, y en la última columna se establece el grupo al que pertenece el índice de capacidad propuesto. La M significa multivariado.

Tabla1

Resumen de ICPM encontrados en la literatura en el periodo 1991-2014

\begin{tabular}{lllcc}
\hline Año & Autor & Índice & DNM & Grupo \\
\hline 1991 & Humbele et al. & C $_{\mathrm{pm}}$ & Sí & 1 \\
1993 & Taam y Liddy & MC $_{\mathrm{PM}}$ & Sí & 1 \\
1994 & Chen & MCp & Sí & 1 \\
1995 & Shahriari y Lawrence & MPCV & Sí & 4 \\
1998 & Wang y Chen & MCp, MCpk, MCpm y & Sí & 1 y 3 \\
2000 & Wang y Du & MCpmk & Sí & 1 y 3 \\
2001 & Yeh y Chen & MCp y MCpc & No & 2 \\
2005 & Castagliola y Castellanos & MCf & Sí & 2 \\
2005 & Wang & MCp y MCpk & Sí & 1 y 3 \\
2006 & Wang & MCpc & No & 4 \\
2007 & Pearn, Wang y Yen & MCp & Sí & 1 \\
2008 & Castagliola y Castellanos & BCp y BCpk & No & 2 \\
\hline & & & & (continúa)
\end{tabular}


(continuación)

\begin{tabular}{|c|c|c|c|c|}
\hline Año & Autor & Índice & DNM & Grupo \\
\hline 2009 & Shahriari y Abdollahzadeh & NMPCV & Sí & 1 \\
\hline 2009 & $\begin{array}{l}\text { Ahmad, Abdollahian, } \\
\text { Zeephongsekul y Abbasi }\end{array}$ & $\mathrm{PNC}_{\text {Total }}$ & No & 2 \\
\hline 2009 & González y Sánchez & $\mathrm{C}_{\mathrm{n}}^{\mathrm{s}}$ & Sí y No & 2 у 3 \\
\hline 2009 & Shinde y Khadse & Mp1 y Mp2 & Sí & 2 y 3 \\
\hline 2010 & Pan y Lee & NMCpm & Sí & 1 \\
\hline 2011 & Goethals y Cho & MCpmc & Sí & 4 \\
\hline 2012 & Hamadani & MCPLs & Sí & 2 y 3 \\
\hline 2013 & Cuamea, Anaya y Luna & $\begin{array}{l}\text { Extensiones de Cpm y } \\
\text { Cpmk }\end{array}$ & Sí & 1 y 2 \\
\hline 2014 & Kováríky Sarga & $\mathrm{C}_{\mathrm{s}}$ & No & 1 \\
\hline 2014 & Cuamea y Rodríguez & $C_{p M}$ y $C_{p k M}$ & Sí & 1 \\
\hline
\end{tabular}

Fuente: Cuamea, G. y Rodriguez, M. (2014)

Los ICPM, en general, pueden ser obtenidos de tres maneras: (a) desde la proporción de una región de tolerancia a una región de proceso; (b) al calcular la probabilidad de productos que no cumplen con las reglas; y (c) por los enfoques en los que las funciones de pérdida son empleadas.

Una manera adicional es nuestra propuesta, que se basa en el siguiente enunciado de Cuamea (et al., 2009):

En el estudio multivariado, las variables críticas deben estar dentro de sus respectivas especificaciones para que el producto se considere aceptable. Si solo una de ellas no cumple con las especificaciones esto será suficiente para que el cliente considere inaceptable el producto.

Sobre la base de esto, para el caso multivariado, se hace el estudio univariado a cada variable de datos normales, y concluimos, respecto al caso multivariado, usando un índice propuesto en este trabajo.

\section{2. ÍNDICE DE CAPACIDAD DE PROCESOS MULTIVARIADOS}

$\mathrm{Al}$ citar algunos autores de la tabla 1, se tiene: Humbele (et al., 1991), usando la distribución multivariada normal, define ICPM como la 
razón de una región de tolerancia rectangular y una región de tolerancia modificada, que es el rectángulo más pequeño alrededor de la elipse con un error especificado de tipo I $(\alpha=0,0027)$. El número de las variables de calidad en el proceso es tenido en cuenta tomando la $v$-ésima raíz de la proporción, en la que representa el número de variables del proceso en estudio.

$$
C_{P M}=\sqrt[v]{\frac{\text { Volumen de la región de tolerancia de ingeniería }}{\text { Volumen de la región de proceso de ingeniería }}}
$$

Taam (et al., 1993) define un índice de capacidad multivariado $\left(\mathrm{MC}_{\mathrm{PM}}\right)$ como la razón del volumen de la región de tolerancia modificada y el volumen de la escala 99,73 \% de la región de proceso. La región de tolerancia modificada es definida como la elipse más grande centrada en el blanco totalmente ubicado dentro de la región de tolerancia original. Luego, para ajustar el cálculo estimado del índice de capacidad normal multivariado, se usó:

$$
\widehat{\mathrm{MC}}_{\mathrm{PM}}=\frac{\mathrm{C}_{\mathrm{PM}}}{\hat{\mathrm{D}}}
$$

donde $\widehat{D}=\sqrt{1+\frac{n}{n-1}\left(\overline{\boldsymbol{X}}-\boldsymbol{\mu}_{0}\right) \boldsymbol{S}^{-1}\left(\overline{\boldsymbol{X}}-\boldsymbol{\mu}_{0}\right)^{T}} . \overline{\mathbf{X}}$ es el vector media muestral, $\mathbf{S}$ es la matriz de varianza-covarianza muestral, y $\mu_{0}$ es la media objetivo del proceso.

Wang (et al., 2000) comparó los ICPM mencionados anteriormente y presentó algunos ejemplos gráficos para ilustrarlos. Sin embargo, la correlación entre las variables no fue tenida en cuenta. Ellos estiman tanto en conjunto como individualmente los índices de capacidad de proceso para los datos multivariados (sin la correlación). Castagliolia y Castellanos (2005) prolongaron el método univariado de Castagliolia a la distribución multivariada, reemplazando la función de densidad de probabilidad univariado $f(x)$ con su versión multivariada, mientras que Wang y Humbele (2000) y Wang y Du (2000) propusieron un método que utiliza el análisis de componentes principales para describir el comportamiento de un proceso para datos multivariados. La ventaja de este enfoque radica en que el análisis de componentes principales es capaz no solo de reducir la dimensión del problema, sino también de transformar datos correlacionados en independientes, lo que simplifica 
considerablemente los cálculos. Cuamea y Anaya proponen los índices $\mathrm{C}_{\mathrm{pM}}$ y $\mathrm{C}_{\mathrm{pkM}}$ para una distribución normal multivariada para variables múltiples con correlación, sin embargo, el $\mathrm{C}_{\mathrm{pM}}$ puede usarse para variables sin correlación.

Wang (2006) también usa la distancia geométrica en procesos no normal y normal multivariado. Yeh y Chen (2001) aplican métodos no paramétricos para calcular proporciones no ajustadas y el índice de capacidad de proceso multivariado para procesos no normales.

El paquete estadístico Statgraphics usa el ICPM, $M C P=\frac{Z}{K / 2}$,

donde $Z$ es el valor de la variable aleatoria normal estándar correspondiente a los DPM calculados. DPM es el índice de capacidad defecto por millón, que representa el número estimado de las muestras por millón que estarían fuera de la especificación en una o más variables. $K$ es el múltiplo del sigma especificado en una tabla. En general, $\mathrm{K}=6 . \mathrm{MCP}$ se interpreta similarmente a $\mathrm{C}_{\mathrm{pk}}$ caso univariado. Así, un $M C P \geq 1,33$ es el deseado.

\section{MATERIALES Y MÉTODOS}

Dado un conjunto de $v$ variables obtenidas de un proceso de muestreo, se obtiene un conjunto de datos modelados por una distribución normal, de la cual surgen las estimaciones de los parámetros para determinar el ICPM que va a determinar la capacidad del proceso de producción. Como un requisito, se debe contar con especificaciones para cada una de las $v$ variables de calidad y con estimaciones de los parámetros del proceso, tales como un vector de medias y una matriz de varianzas covarianzas. Posteriormente, las especificaciones fueron utilizadas para definir la región de especificación y, por otro lado, la estimación de los parámetros del proceso se utiliza para crear una región de variación del proceso utilizando los límites de tolerancia natural del proceso.

Para el caso univariado, se tiene una especificación inferior y otra superior para el control de la variable de calidad $X_{i}$, para algún $i=1, \ldots, v$. El objetivo que se persigue es que: $\left(L E I_{i} \leq X_{i} \leq L E S_{i}\right)$, donde $L E I_{i}$ y $L E S_{i}$ son los límites de especificación inferior y superior establecidos para cada variable $X_{i}$. Para el caso multivariado, lo anterior alcanzaría para extenderse y el intervalo para ser reemplazado 
por una región de especificación; dicha región de especificación estará construida por los intervalos de especificación para cada una de las variables de calidad. La región de especificación sería representada mediante las desigualdades:

$$
I_{i=1}^{v}\left(L E I_{i} \leq X_{i} \leq L E S_{i}\right)
$$

Ahora, para el caso normal multivariado se tiene nuestra siguiente propuesta en estudio: Sean $X_{1}, X_{2}, \ldots, X_{v} ; v$ variables aleatorias obtenidas de un proceso, modeladas por una función de distribución multivariada normal, de donde se obtienen las estimaciones de los parámetros para el cálculo de cada ICP para cada $X_{i}, i=1, \ldots, v$. Obtenido cada ICP para cada $X_{i}, i=1, \ldots, v$, se procede a determinar nuestro índice multivariado CPME.

Se usará un ejemplo real en el que las variables están correlacionadas y no correlacionadas, para que a través del modelo se realicen comparaciones de los resultados que se han obtenido de los métodos multivariados empleados por otros autores. Para esto se usarán programas informáticos como el software Matlab 7.6.0, Excel, Minitab V. 17 y Statgraphics Centurión, para obtener algunos de los diferentes índices expuestos anteriormente.

\section{PROPOSICIÓN DE ÍNDICE DE CAPACIDAD PARA USO EN CASO DE VARIABLES MÚLTIPLES}

Dado un conjunto de $v$ variables obtenidas de un proceso de muestreo, se obtiene un conjunto de datos modelados por una distribución normal o no normal, de la cual se obtienen las estimaciones de los parámetros para hallar el ICPM que va a determinar la capacidad del proceso de producción. Cuando los datos no son normales, entonces el índice multivariado requiere de una nueva metodología. En función de esto, en este trabajo se evaluará el desempeño de los métodos de estimación de capacidad existentes para datos con variables de calidad multivariados normal.

Sea $X=\left(X_{1}, X_{2}, \ldots, X_{v}\right)^{\prime}$ el vector de las variables de calidad del proceso.

Sea $\mu=\left(\mu_{1}, \mu_{2}, \ldots, \mu_{v}\right)^{\prime}$ el vector de medias y $\Sigma$ su matriz de covarianzas. 
Sea $S=\left\{X \in \mathbb{R}^{\mathbf{v}}: L E I_{i} \leq X \leq L E S_{i}\right\} i=1, \ldots, v$ la región de tolerancias del proceso.

Se obtienen las estimaciones de los parámetros para el cálculo de cada ICP para cada $X_{i}, i=1, \ldots, v$.

Proponemos el ICPM, definido de la siguiente manera:

$$
\chi_{A_{i}}\left(I c p_{i}\right)=\left\{\begin{array}{lll}
1 & \text { si } & \text { Icp } \\
0 & \text { si } & \text { Icp } \\
\text { Ic } & \notin A_{i}
\end{array}\right.
$$

Donde $A_{i}=\operatorname{Rango}\left(I c p_{i}\right)=\left\{\frac{I c p_{i}}{I c p_{i}} \geq 1,33\right\}$ para cada $X_{i}, i=1, \ldots, v \mathrm{y}$ $I c p_{i}$ : es un índice de capacidad univariado seleccionado.

Definimos el siguiente ICPM: CPME $=\frac{\sum_{i=1}^{v} \chi_{A_{i}}\left(I c p_{i}\right)}{v}$, donde CPME significa: capacidad de proceso multivariado estadístico.

Ahora bien, por definición de CPME se tiene que:

Si $C P M E=1$, entonces el proceso de producción es estable, ya que todas las variables están en el rango de especificación dado.

Si $C P M E \neq 1$, entonces el proceso de producción no es estable, ya que existe al menos una $X_{i}, i=1, \ldots, v$ que esta fuera del rango de especificación dado para cada $X_{i}, i=1, \ldots, v$.

\section{RESULTADOS}

En las siguientes secciones se muestra el desarrollo del índice de capacidad propuesto en este trabajo, tanto con variables normales correlacionadas y no correlacionadas, así como otros índices.

\subsection{Cálculo de CPME y comparación con otros índices propuestos}

En el trabajo de Chen (1994) se discuten dos ejemplos numéricos, en los que se aplicaron tres de los índices de capacidad que aparecen en la literatura. En este trabajo se usan los datos y se trabaja con dos casos: variables correlacionadas y no correlacionadas. Usando los datos del primer ejemplo, se calcula el índice de capacidad propuesto en este 
trabajo y se compara con los índices obtenidos por tres propuestas; el resumen se presenta en la tabla 2 . El ejemplo trata de una distribución normal bivariada, en la cual la dureza Brinell $(\mathrm{H})$ y la resistencia a la tensión (S) son dos variables de calidad de un producto industrial. Las tolerancias de ingeniería para ambas variables de calidad vienen dadas por $[112,3 ; 241,3]$ y [32,7; 73,3], respectivamente, y el vector de valores nominales para $\mathrm{H}$ y $\mathrm{S}$ viene dada por $[177,53]$.

\subsection{Caso variables con correlación}

Después de realizar la colección de 25 mediciones de ambas variables de calidad, se obtuvieron los valores de la tabla 1 . Al realizar la prueba de Shapiro-Wilk, encontramos que las 25 mediciones siguen una distribución normal multivariada con el siguiente vector de medias $[177,52 ; 52,188]$ y la siguiente matriz de varianzas covarianzas:

\begin{tabular}{|c|c|c|}
\hline & $\mathrm{H}$ & $\mathrm{S}$ \\
\hline $\mathrm{H}$ & 329,093 & 91,8898 \\
\hline & $(25)$ & $(25)$ \\
\hline $\mathrm{S}$ & 91,8898 & 38,8653 \\
\hline & $(25)$ & $(25)$ \\
\hline
\end{tabular}

Tabla 2

Dureza Brinell (H) y resistencia a la tensión (S) para un producto industrial

\begin{tabular}{cccccc}
\hline $\mathbf{H}$ & $\mathbf{S}$ & $\mathbf{H}$ & $\boldsymbol{S}$ & $\boldsymbol{H}$ & $\boldsymbol{S}$ \\
\hline 143 & 31,0 & 141 & 47,3 & 178 & 50,9 \\
200 & 57,0 & 175 & 57,3 & 196 & 57,9 \\
168 & 47,5 & 187 & 58,5 & 160 & 45,5 \\
181 & 53,4 & 187 & 58,2 & 183 & 53,9 \\
148 & 47,8 & 186 & 57,0 & 179 & 51,2 \\
178 & 51,5 & 172 & 49,4 & 194 & 57,5 \\
162 & 45,9 & 182 & 57,2 & 181 & 55,6 \\
215 & 59,1 & 177 & 50,6 & & \\
161 & 48,4 & 204 & 55,1 & & \\
\hline
\end{tabular}

Fuente: Cuamea, G. y Rodriguez, M. (2014)

Con un $p>0,150$, se concluye, según la prueba KolmogorovSmirnov, que H y S siguen una distribución normal. KS $=0,129$ y $\mathrm{KS}=0,140$ para $\mathrm{H}$ y $\mathrm{S}$, respectivamente. 
Capacidad de procesos para $\mathrm{H}$

$\mathrm{LIE}=32,7$, nominal $=53,0, \mathrm{LSE}=73,3$

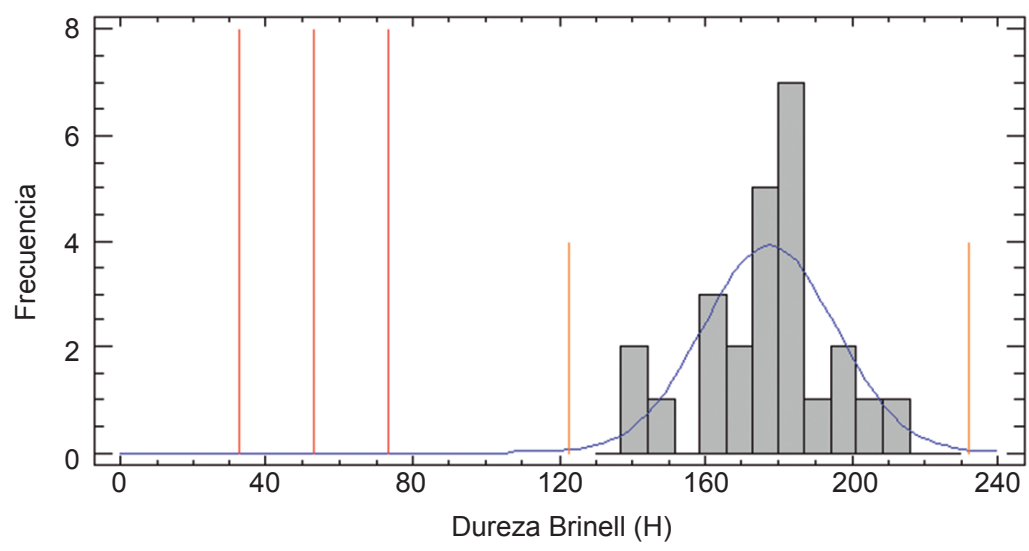

Normal

Media $=177,52$

Desv. est. $=18,1409$

Capacidad de proceso para S

$\mathrm{LIE}=112,3$, nominal $=177,0, \mathrm{LSE}=241,3$

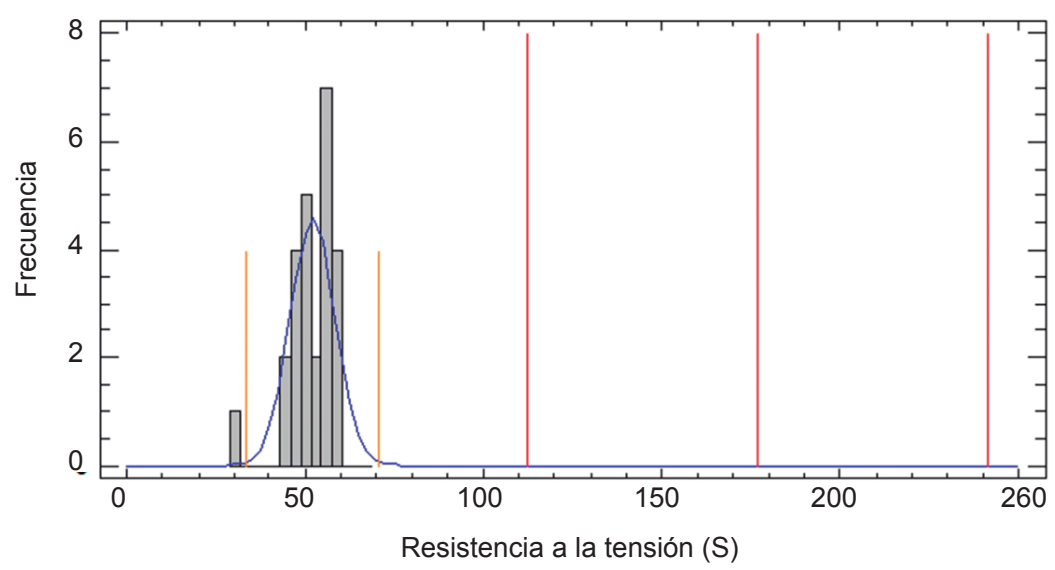

Normal

Media $=52,188$

Desv. est. $=6,2342$

Figura 1. Graficas de capacidad del proceso para la dureza $(H)$ y la resistencia a la tensión $(S)$. Caso variables correlacionadas

Elaboración propia 
Tabla 3

Índices de capacidad univariado para las variables $\mathrm{H}$ y $\mathrm{S}$

\begin{tabular}{cccc}
\hline Variable & $\begin{array}{c}\text { Índice de } \\
\text { capacidad }\end{array}$ & $\begin{array}{r}\text { Capacidad } \\
\text { (dentro de) }\end{array}$ & Capacidad general \\
\hline $\mathrm{H}$ & $C_{p} / P_{p}$ & 1,06602 & 1,18517 \\
$C_{p k} / P_{p} K$ & 1,05412 & 1,17194 \\
$C_{p m}$ & & 1,18 \\
& $C_{p} / P_{p}$ & 1,1211 & 1,08541 \\
& $C_{p k} / P_{p} K$ & 1,07625 & 1,04199 \\
& $C_{p m}$ & & 1,16 \\
\hline
\end{tabular}

Elaboración propia

Para realizar el cálculo del índice de capacidad potencial que se propone en este trabajo, ubicaremos el promedio del proceso en el centro de las especificaciones.

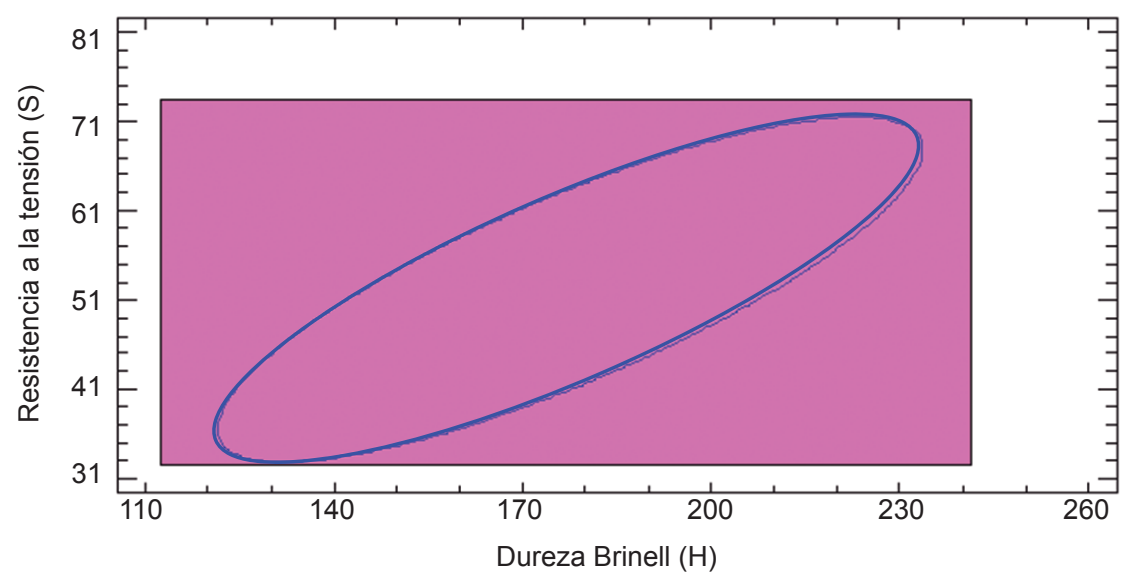

Figura 2. Elipse de capacidad del proceso para la dureza $(H)(a)$ y la resistencia a la tensión $(\mathrm{S})(\mathrm{b})$. Caso variables correlacionadas

Elaboración propia 
Tomando $I c p_{i}=C_{p}$, entonces: $C P M E=\frac{\sum_{i=1}^{v} \chi_{A_{i}}\left(C_{p}\right)}{v}=\frac{0+0}{2}=0$, así $C P M E\left(C_{p}\right)<1$

De forma análoga, se obtienen los resultados que se muestran en la tabla 4 .

En la gráfica de la figura 2 se observa que el proceso se sale ligeramente de la región de especificación, lo que deberá ser reflejado por el cálculo del CPME, el cual, para este proceso, deberá tener un valor de índice menor que 1.

Tabla 4

Comparación de varios índices de capacidad para dos variables de calidad con correlación

\begin{tabular}{|c|c|c|c|c|}
\hline \multicolumn{2}{|c|}{$\begin{array}{l}\text { Taam et al. } \\
(1993)\end{array}$} & $\begin{array}{c}\text { Índice de Cuamea } \\
\text { y Rodríguez } \\
(2014)\end{array}$ & $\begin{array}{c}\text { Índice de } \\
\text { Statgraphics } \\
(2016)\end{array}$ & $\begin{array}{c}\text { Índice de Salazar } \\
\text { y Fermín } \\
(2016)\end{array}$ \\
\hline \multirow[t]{2}{*}{$\widehat{M C}_{P}$} & \multirow{2}{*}{$\widehat{M C}_{P M}$} & \multirow[t]{2}{*}{$C_{p M}$} & \multirow[t]{2}{*}{$M C P$} & $C P M E\left(P_{p}\right)$ \\
\hline & & & & $C P M E\left(C_{p m}\right)$ \\
\hline 1,71 & 1,65 & 0,95 & 1,05 & $<1$ \\
\hline
\end{tabular}

Elaboración propia

\subsection{Caso variables no correlacionadas}

Después de efectuar 25 mediciones de ambas variables de calidad, se obtuvieron los valores de la tabla 4. Al realizar la prueba de ShapiroWilk, se encontró que las 25 mediciones siguen una distribución normal multivariada con el siguiente vector de medias [177,106; 53,157] y la siguiente matriz de varianzas covarianzas:

\begin{tabular}{|c|c|c|}
\hline & $\mathbf{H}$ & $\mathbf{S}$ \\
\hline $\mathbf{H}$ & 0,765983 & $-0,0296604$ \\
\hline & $(25)$ & $(25)$ \\
\hline $\mathbf{S}$ & $-0,0296604$ & 1,09009 \\
\hline & $(25)$ & $(25)$ \\
\hline
\end{tabular}


Tabla 5

Dureza Brinell $(H)$ y resistencia a la tensión (S) para un producto industrial

\begin{tabular}{cccccc}
\hline $\mathbf{H}$ & $\mathbf{S}$ & $\mathbf{H}$ & $\mathbf{S}$ & $\mathbf{H}$ & $\mathbf{S}$ \\
\hline 176,437 & 53,5317 & 175,588 & 52,3967 & 176,818 & 54,1309 \\
176,596 & 54,7648 & 176,807 & 52,1516 & 176,800 & 51,9326 \\
176,092 & 51,7810 & 177,441 & 52,7447 & 176,051 & 54,0050 \\
176,566 & 53,5707 & 176,926 & 52,0609 & 178,095 & 51,8588 \\
176,940 & 53,1057 & 177,540 & 54,6906 & 178,384 & 53,9194 \\
178,321 & 53,8484 & 177,634 & 53,5954 & 179,515 & 52,6633 \\
177,161 & 52,4804 & 176,789 & 52,2339 & 177,645 & 52,7505 \\
177,128 & 53,0898 & 175,812 & 54,4680 & & \\
177,209 & 55,3306 & 177,357 & 51,8202 & & \\
\hline
\end{tabular}

Elaboración propia

Con un $\mathrm{p}>0,150$, se concluye, según la prueba Kolmogorov-Smirnov, que para $\mathrm{H}$ y $\mathrm{S}$ no hay evidencia suficiente que sugiera que los datos no siguen una distribución normal KS $=0,109$ y KS $=0,131$ para $\mathrm{H}$ y $\mathrm{S}$, respectivamente.

Tabla 6

Índices de capacidad univariado para las variables $\mathrm{H}$ y $\mathrm{S}$

\begin{tabular}{cccc}
\hline Variable & Índice de capacidad & $\begin{array}{c}\text { Capacidad } \\
\text { (dentro de) }\end{array}$ & Capacidad general \\
\hline $\mathbf{H}$ & $C_{p} / P_{p}$ & 24,33 & 24,37 \\
$C_{p k} / P_{p} k$ & 24,22 & 24,45 \\
$C_{p m}$ & & 24,30 \\
$\mathbf{S}$ & $C_{p} / P_{p}$ & 6,48 \\
$C_{p k} / P_{p} k$ & 6,34 & 6,43 \\
$C_{p m}$ & 6,29 & 6,41 \\
\hline $\begin{array}{l}\text { Correlación } \\
\text { de Pearson }\end{array}$ & & \\
\hline
\end{tabular}

Elaboración propia 
Capacidad de proceso para $\mathrm{H}$

LIE $=112,3$, nominal $=177,0$, LSE $=241,3$

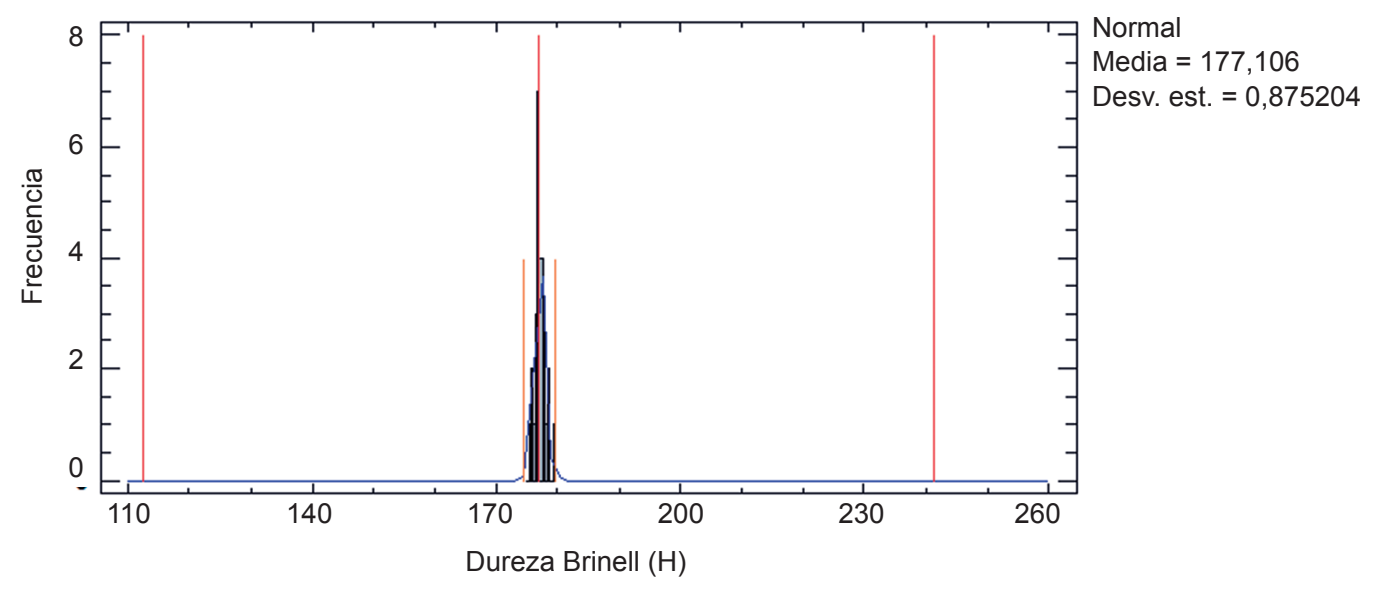

Capacidad de proceso para $\mathrm{S}$

$\mathrm{LIE}=32,7$, nominal $=53,0, \mathrm{LSE}=73,3$

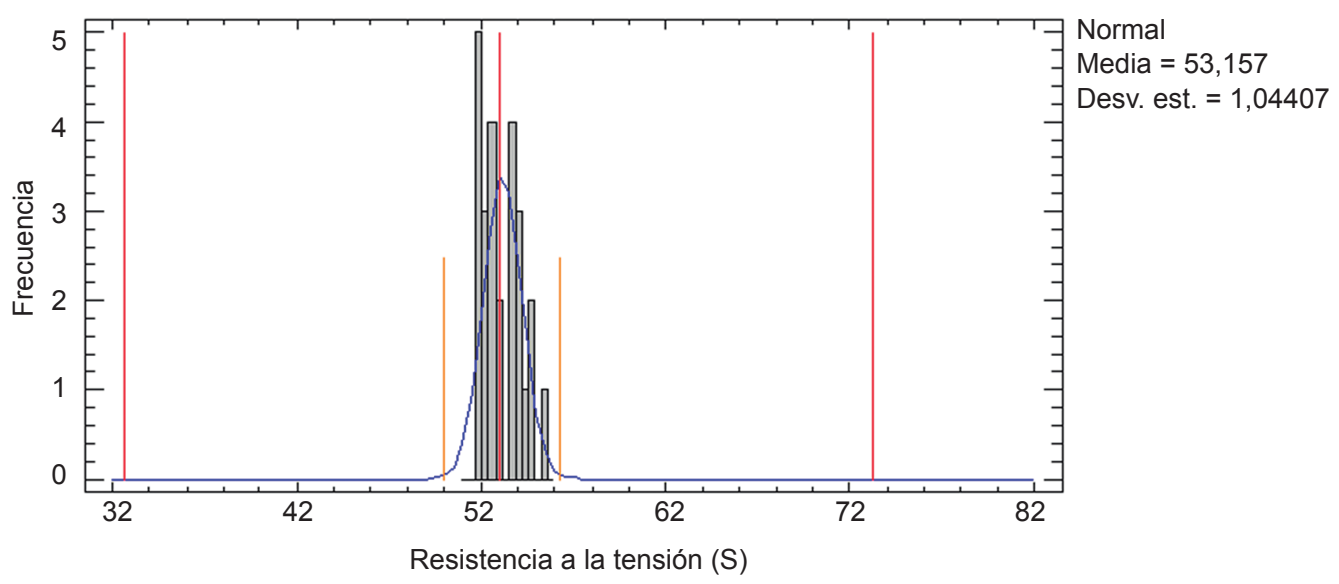

Figura 3. Gráficas de capacidad del proceso para la dureza $(H)(c)$ y la resistencia a la tensión $(\mathrm{S})(\mathrm{d})$. Caso sin correlación

Elaboración propia 


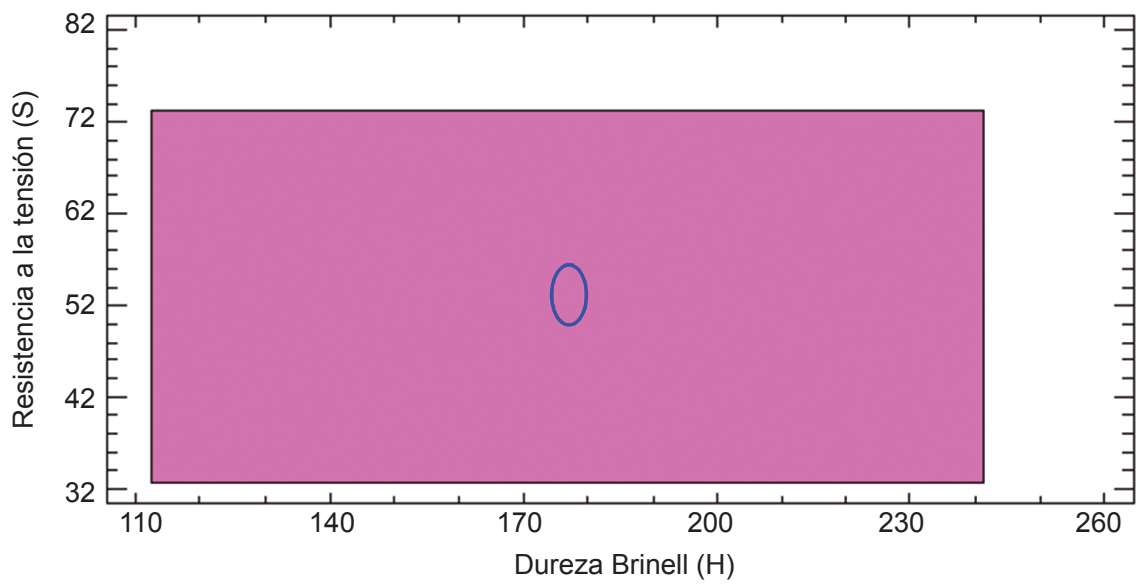

Figura 4. Elipse de capacidad del proceso para la dureza $(\mathrm{H})$ y la resistencia a la tensión (S) Elaboración propia

En la figura 4 se observa que el proceso está totalmente contenido en la región de especificación, lo que deberá ser reflejado por el cálculo del ICPME, el cual deberá tener un valor de este índice igual que 1 para este proceso.

Tabla 7

Comparación de varios índices de capacidad para dos variables de calidad sin correlación

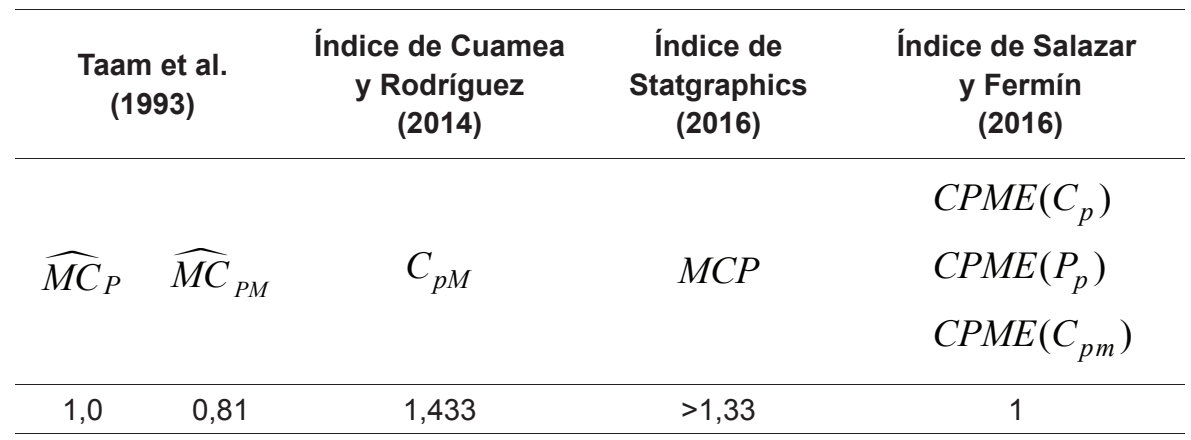

Elaboración propia 


\section{DISCUSIÓN}

Para los casos estudiados de variables correlacionadas y no correlacionadas, las variables pueden modelarse a través de una distribución normal multivariada, y en ambos casos el cálculo del índice de capacidad CPME, resulta simple y fácil. Su interpretación va acorde con la situación presentada por los datos, además que es aplicable para cualquier dimensión. En el primer caso, la figura 2 muestra que el proceso se sale ligeramente de la región de especificación, que es reflejado por el índice $C P M E$, el cual indica que en este caso la capacidad de proceso bivariable $(\mathrm{H}, \mathrm{S})$ no es estable. Sin embargo, para el siguiente caso la figura 4 muestra que el proceso está totalmente contenido en la región de especificación, lo cual es reflejado por el cálculo del CPME, el que indica que la capacidad de proceso bivariable $(\mathrm{H}, \mathrm{S})$ es estable. La ventaja que presenta el CPME es que puede ser usado en ambos casos de variables con correlación y sin correlación, no requiere de cálculos complejos y es de fácil interpretación.

\section{REFERENCIAS}

Ahmad, S., Abdollahian, M., Zeephongsekul, P. y Abbasi, B. (2009). Multivariate non normal process capability analysis. International Journal of Advanced Manufacturing Technology, 4(7), 757-765.

Bothe, D.R. (1999). Composite Capability index for multiple product characteristics. Quality Engineering, 12(2), 253-258.

Castagliola, P. y Castellanos, J.V.G. (2008). Process capability indices dedicated to bivariate non normal distributions. Journal of Quality in Maintenance Engineering, 14(1), 87-101.

Castagliola, P. y Castellanos, J.V.G. (2005). Capability Indices Dedicated to the Two Quality Characteristics Case. QualityTechnology and Quantitative Management, 2(2), 201-220.

Cuamea, G. y Rodriguez, M. (2014). Propuesta para evaluar la capacidad de procesos de manufactura multivariados. Revista Ingeniería Industrial, 2, 35-47.

Cuamea, G. y Anaya, C. (2009). Evaluación de la calidad en productos o procesos con múltiples características de calidad correlacionadas. Epistemus, 6, 11-16. 
Foster, E.J., Barton, R.R. y Gautam, N. (2005). The process-oriented multivariate capability index. International Journal of Production Research, 43(10), 2135-2148.

Goethals, P.L. y Cho, B.R. (2011).The development of a target-focused process capability index with multiple characteristics. Quality and Reliability Engineering International, 27(3), 297-311.

González, I. y Sanchez, I. (2009). Capability indices and nonconforming proportion in univariate and multivariate process. The International Journal of Advanced Manufacturing Technology, 44(9), 1036-1050.

Kane, V.E. (1986). Process capability indices. Journal of Quality Technology, 34(1), 1-19.

Kotz, S. y Johnson, N.L. (2002). Process Capability Indices-a Review, 1992-2000. Journal of Quality Technology, 34(1), 2-19.

Taam, W. y Liddy, J.W. (1993). A Note of Multivariate Capability Indices. Journal of Appllied Statistics, 20(3), 339-351. 
